J Ästhet Chir 2020 · 13:78

https://doi.org/10.1007/s12631-020-00225-x

Online publiziert: 4. Mai 2020

(c) Springer Medizin Verlag $\mathrm{GmbH}$, ein Teil von Springer Nature 2020

Dirk Brandl

Drensteinfurt, Deutschland

\title{
Erwiderung zum Leserbrief zum Beitrag „Fotodokumentation ästhetischer Behandlungen“
}

\section{Erwiderung}

Zum Leserbrief von Löw O (2020) Publiziertes Konzept von M. Siessegger zur standardisierten Fotodokumentation ästhetischer Behandlungen (2012) fand keine Erwähnung. J Ästhet Chir 13. https://doi.org/10.1007/s12631-02000224-y

\section{Originalbeitrag}

Brandl D, Prantl L (2019) Fotodokumentation ästhetischer Behandlungen. Vorschläge zur Standardisierung der Fotodokumentation in der ästhetischen Medizin. J Ästhet Chir 12:84-94. https://doi.org/10.1007/s12631019-0180-9.

Sehr geehrte Redaktion, lieber Herr Dr. Dr. Siessegger,

Ihren von einer Marketingagentur verfassten Leserbrief haben wir zur Kenntnis genommen. Die von uns vorgeschlagene Standardisierung wurde erstmals 2007 öffentlich von dem Diplomfotoingenieur Dirk Brandl und dem Chirurgen und Dermatologen Dr. Klaus Eichhorn in einem Seminar der MASTER Conference des NETZWERK-Lipolyse vorgestellt und im Laufe der Jahre immer weiter verfeinert. Zeugnis davon sind weitere Seminare in 2008 und 2009 sowie erste Veröffentlichungen $\mathrm{zu}$ dem Thema in 2013 (Kosmetische Medizin 6-13), dem 2013 erschienenen E-Book von Dirk Brandl „Fotodokumentation in der Ästhetischen Medizin und Dermatologie" (Apple iBook Store), 2015 und 2016 (Haut 05-15, 06-15, 01-16), und letztendlich 2017 in der Plastic and Reconstructice Surgery Global Open.
Die in die Nähe eines Plagiates gesetzten Bilder - ein Vorwurf, gegen den wir uns auf das Schärfste verwehren - sind in ihrer Struktur identisch mit den von di Bernardo et al. verwendeten 5 Ansichten, also weder von uns noch von Herrn Dr. Siessegger ursprünglich vorgeschlagen. Dies wurde auch in dem Artikel als Ursprung zitiert.

Das Buch von Herrn Dr. Siessegger war uns bis zu diesem Leserbrief nicht bekannt, deshalb kann es weder zitiert werden noch eine inhaltliche Auseinandersetzung stattfinden (PubMed Listung nicht vorhanden).

Mit freundlichen Grüßen

Dirk Brandl

im Namen der Autoren

\section{Korrespondenzadresse}

Dipl.-Ing. Dirk Brandl

Mühlenstr. 19, 48317 Drensteinfurt, Deutschland

brandl@network-globalhealth.com

Interessenkonflikt. D. Brandl gibt an, dass kein Interessenkonflikt besteht. 